\title{
The Type VI Secretion System: A Multipurpose Delivery System with a Phage-Like Machinery
}

\author{
Angela R. Records \\ Department of Cell Biology \& Molecular Genetics, University of Maryland, College Park, MD 20742, U.S.A.
}

Submitted 14 November 2010. Accepted 22 February 2011.

\begin{abstract}
Whether they live in the soil, drift in the ocean, survive in the lungs of human hosts or reside on the surfaces of leaves, all bacteria must cope with an array of environmental stressors. Bacteria have evolved an impressive suite of protein secretion systems that enable their survival in hostile environments and facilitate colonization of eukaryotic hosts. Collectively, gram-negative bacteria produce six distinct secretion systems that deliver proteins to the extracellular milieu or directly into the cytosol of host cells. The type VI secretion system (T6SS) was discovered recently and is encoded in at least one fourth of all sequenced gramnegative bacterial genomes. T6SS proteins are evolutionarily and structurally related to phage proteins, and it is likely that the T6SS apparatus is reminiscent of phage injection machinery. Most studies of T6SS function have been conducted in the context of host-pathogen interactions. However, the totality of data suggests that the T6SS is a versatile tool with roles in virulence, symbiosis, interbacterial interactions, and antipathogenesis. This review gives a brief history of T6SS discovery and an overview of the pathway's predicted structure and function. Special attention is paid to research addressing the T6SS of plant-associated bacteria, including pathogens, symbionts and plant growth-promoting rhizobacteria.
\end{abstract}

Bacteria inhabit virtually every environment on earth. In order to cope with the many stressors associated with a diversity of habitats, bacteria have evolved an arsenal of survival tools, including a variety of protein secretion systems. Until recently, five distinct protein secretion systems had been identified in gram-negative bacteria. These secretion pathways, types I to V, facilitate the transport of chemical messages from the bacterial cytoplasm to the outside of the cell or, in some cases, directly into the cells of a eukaryotic host. Bacterial protein secretion systems can be broadly categorized as either one- or two-step. One-step secretion systems, including types I, III, and IV, serve as direct conduits through the bacterial cell envelope. The type I secretion system (T1SS) is a common and relatively simple machine composed of just three proteins-an outer membrane protein (OMP), an inner membrane ATP-binding cassette $(\mathrm{ABC})$ transporter, and a membrane fusion protein that spans the periplasm and connects the OMP and ABC components (Delepelaire 2004). T1SS substrates, usually enzymes or toxins, are targeted to the secretion apparatus by $\mathrm{C}$-terminal secretion signals and are then secreted out of the bacterial cell in a single step (Duong et al. 1996). A wide range of gram-

Corresponding author: A. R. Records; E-mail: angela.records@gmail.com negative pathogens and symbionts utilize the type III secretion system (T3SS) to inject effector proteins directly into eukaryotic host cells. The T3SS consists of more than 20 proteins-many with homology to flagellum assembly machinery-that function together as a nanosyringe. T3SS effectors are targeted to the secretion apparatus by N-terminal secretion signals and are delivered, in one step, through both the bacterial cell wall and the eukaryotic plasma membrane (Cornelis 2006). The Type IV secretion system (T4SS) is arguably the most versatile of the secretion pathways, with substrates ranging from monomeric proteins to multi-subunit protein toxins to nucleoprotein complexes. Functional T4SS have been identified in gramnegative, gram-positive, and wall-less bacteria, as well as in certain Archaea spp. (Alvarez-Martinez and Christie 2009). Several pathogenic bacteria use a T4SS to target proteins or single-stranded DNA-protein complexes into host cells. Substrate targeting to the T4SS is complex and may be mediated by a combination of factors, including C-terminal secretion signals and additional intrinsic motifs (Alvarez-Martinez and Christie 2009). The multiprotein T4SS is structurally and evolutionarily related to conjugation machinery.

Two-step secretion systems require that a protein substrate be first translocated across the inner membrane via either the general secretory pathway (the Sec translocon) or the twinarginine translocation (Tat) pathway. After Sec and Tat substrates arrive in the periplasm, their $\mathrm{N}$-terminal signal sequences are cleaved and the proteins are then delivered to the extracellular milieu in a second step. The two-step type II secretion system (T2SS) consists of a set of twelve core components that provide a pathway from the periplasm through the outer membrane to the outside of the bacterial cell (Cianciotto 2005). T2SS are broadly conserved and are often employed by pathogens to secrete exoenzyme virulence factors. The type V autotransporter pathway (T5SS) is also a widely distributed, twostep pathway used in the secretion of exoenzymes. The T5SS is unique in that it is synthesized as a single polypeptide with an effector-like "passenger" domain and a C-terminal "transporter" domain. Following delivery into the periplasm by the Sec translocon, T5SS substrates mediate their own transport outside of the cell by forming $\beta$ barrels in the outer membrane, through which they autotransport their passenger domains (Henderson et al. 2004).

\section{Identification of a sixth secretion pathway: clues from Rhizobium spp.}

In 2006, members of the Mekalanos laboratory described a novel protein-secretion system elaborated by the human pathogens Pseudomonas aeruginosa and Vibrio cholerae (Mougous et al. 2006; Pukatzki et al. 2006). Critical findings that led to 
the identification of the type VI secretion system (T6SS) can be traced to 1997, when Roest and colleagues (1997) identified a Rhizobium leguminosarum bv. trifolii genetic locus that inhibits the symbiont's ability to effectively nodulate pea. Sequencing of the "imp" locus (impaired in nodulation) revealed no significant homology to any genes known at the time of its discovery. It is clear now, however, that $R$. leguminosarum is one of many plant- and animal-associated bacterial species that carry such gene clusters-similar loci are found in the genomes of a wide range of gram-negative bacteria (Bingle et al. 2008; Boyer et al. 2009). Clues to the function of the imp locus came in 2003, when proteomic analyses of culture supernatants revealed that the $R$. leguminosarum impJ operon is required for the secretion of a $27-\mathrm{kDa}$ protein, $\mathrm{RbsB}$, with an $\mathrm{N}$-terminal signal sequence (Bladergroen et al. 2003). Similar observations were soon made for other organisms, including $V$. cholerae and P. aeruginosa (Mougous et al. 2006; Pukatzki et al. 2006) and the fish pathogen Edwardsiella tarda (Rao et al. 2004). Importantly, secreted proteins associated with the $V$. cholerae and $P$. aeruginosa imp-like loci have no recognizable signal sequences, and the loci contain a set of genes distinct from previously identified protein secretion systems. Indeed, the 2006 characterization of these loci was a seminal discovery (Mougous et al. 2006; Pukatzki et al. 2006). The proteins encoded by the imp locus and its counterparts in other bacteria are now known to make up a T6SS, which appears to have an important role in pathogen-symbiont host interactions. Several of the genes in the T6SS locus have been implicated in virulence activities, including chronic lung infection in rats (Potvin et al. 2003), replication in protozoa (Das et al. 2002), adherence to human intestinal cells (Pukatzki et al. 2006), and actin cross-linking in eukaryotic cells (Pukatzki et al. 2006).

\section{The T6SS machine: repurposed phage parts?}

The first glimpse at a T6SS structural component came from electron microscopy and X-ray crystallography of the Hcp1 protein, which is secreted in abundance by $P$. aeruginosa (Mougous et al. 2006). Interestingly, Hcp (haemolysin coregulated protein) secretion was first noted in 1996, and its lack of a signal sequence hinted at a novel mechanism of protein secretion (Williams et al. 1996). Indeed, Hcp secretion is dependent on other genes in the T6SS locus, and in a study of the $E$. tarda Evp T6SS, systematic deletions revealed that 13 of the 16 evp genes are required for export of the Hcp1-like EvpC protein (Zheng and Leung 2007). Hcp1 was shown to form a hexameric ring structure with external and internal diameters of 8.5 and $4.0 \mathrm{~nm}$, respectively (Jobichen et al. 2010; Mougous et al. 2006). Hcp1 hexamers stack in a head-to-tail fashion; and this observation led to speculation that, rather than functioning as secreted effectors, Hcp1 rings may assemble to form a conduit through which other proteins are delivered to the extracellular milieu. Indeed, Hcp1 hexameric rings can selfassemble into nanotubes in vitro, when disulfide bonds are engineered to stabilize the ring-ring interface (Ballister et al. 2008). Interestingly, the Hcp1 nanotube structure is strikingly similar to the major tail protein of phage $\lambda$ (Pell et al. 2009) and has internal and external diameters that are nearly identical to those of the bacteriophage T4 tail tube (Leiman et al. 2009). In addition to structural similarity, Hcp1 exhibits sequence similarity to T4 gene product (gp) 19, which polymerizes to form the phage tail tube, a channel for DNA delivery into bacterial cells (Leiman et al. 2004, 2009). These structural and sequence similarities strongly suggest that there is an evolutionary relationship between phage tails and the T6SS.

Other proteins encoded in the T6SS locus have homology to bacteriophage parts. Models of the T4 phage structure and a putative T6SS structure are compared in Figure 1, and their relevant components are described below. Like Hcp1, VgrG proteins have been identified in the culture supernatants of several bacterial species (Mattinen et al. 2007; Pukatzki et al. 2006; Zheng and Leung 2007). VgrG proteins are structurally similar to the T4 bacteriophage tail spike proteins gp27 and gp5 (Pukatzki et al. 2007). Proteins gp27 and gp5 form a complex that extends from the phage tail tube and terminates with a sharp tip used by the phage to puncture the bacterial cell surface (Mesyanzhinov et al. 2004). A T6SS protein identified as VCA0109 has sequence homology to T4 gp25, which forms part of the phage tail baseplate (Leiman et al. 2009). The $V$. cholerae T6SS proteins VipA and VipB form a complex of tubular, cogwheel-like structures with internal diameters of $10.0 \mathrm{~nm}$ (Bönemann et al. 2009). The VipA/VipB tubule is similar in structure to the $\mathrm{T} 4$ tail-sheath protein, which encases the viral tail tube and contracts to push the phage needle through the outer membranes of target bacterial cells (Aksyuk et al. 2009). Bönemann and colleagues noted that the internal diameter of a VipA/VipB tubule is of sufficient size to accommodate an 8.5-nm Hcp tube, which could allow VipA/VipB to function like the tail sheath of a contractile phage-pushing the Hcp tube through the bacterial outer membrane from the inside (Bönemann et al. 2010). A recent characterization of the Burkholderia cenocepacia VipA $\left(\mathrm{BcsL}_{\mathrm{B}}\right)$ and $\mathrm{VipB}\left(\mathrm{BcsK}_{\mathrm{C}}\right)$ orthologs is consistent with the findings in V. cholerae (Aubert et al. 2010). Based on the structural similarities between Hcp, VgrG, VCA0109, VipA/VipB, and bacteriophage injection machinery, it has been suggested that the T6SS may appear as an inverted phage tail on the surface of the bacterial cell, where it secretes effector proteins into the extracellular milieu or injects them directly into target cells (Fig. 1) (Bönemann et al. 2010; Cascales 2008; Leiman et al. 2009). Although no evidence has yet been provided, it is also possible that T6SS-like phage are capable of delivering nucleic acid into target cells. The two injection machines share a common evolutionary history (Leiman et al. 2009), and it is likely that many gram-negative bacteria have repurposed proteins encoded by phage genes that were, at some point, integrated into their genomes.

\section{The T6SS locus.}

What constitutes a bona fide T6SS locus? Putative T6SS gene clusters have been identified in the genomes of at least 100 gram-negative bacteria, and comparisons between their sequences have prompted attempts at defining a set of T6SS "core components" (Bingle et al. 2008; Boyer et al. 2009; Zheng and Leung 2007). The typical T6SS locus consists of 15 to 20 conserved open reading frames. T6SS gene clusters vary amongst bacterial genomes in terms of gene order and orientation, but all share a conserved group of 13 essential genes (Boyer et al. 2009; Zheng and Leung 2007). In addition to the five proteins described above, putative functions have been assigned to four other essential proteins encoded in the T6SS locus. The T6SS inner membrane proteins IcmF and DotU are homologous to accessory proteins of the Legionella pneumophila T4SS, in which they interact with one another and are believed to provide structural stability to the T4SS machinery and assist in its assembly (Sexton et al. 2004; VanRheenen et al. 2004). The IcmF and DotU proteins encoded by the T6SS locus also interact with one another, suggesting that they may be structural proteins like their T4SS orthologs (Ma et al. 2009a; Zheng and Leung 2007). In a yeast two-hybrid assay, IcmF interacts with SciN, an outer membrane lipoprotein that is exposed to the periplasm (Aschtgen et al. 2008; Zheng and Leung 2007). Thus, IcmF, DotU, and SciN likely make up structural components of the T6SS that, collectively, span the inner and outer membranes of the bacterial cell (Fig. 1). The IcmF protein possesses a conserved Walker A motif, which is 
characteristic of ATPase proteins. The role of the IcmF Walker A motif is not well-understood, however, because it is important for secretion activity in certain systems but dispensable in others (Ma et al. 2009a; Zheng and Leung 2007). One of the first T6SS genes studied, $\operatorname{clpV}$, encodes an ATPase of the Hsp100/Clp AAA+ family and was initially believed to be responsible for energizing the effector transport process (Mougous et al. 2006; Schlieker et al. 2005). Indeed, the other bacterial secretion systems each have ATPase components with structural similarity to $\mathrm{ClpV}$, which suggests a conserved role for ATPases in protein secretion. ClpV forms a hexameric ring that may sit at the base of the T6SS apparatus and deliver unfolded protein effectors to the Hcp nanotube. Alternatively, $\mathrm{ClpV}$ may be involved in T6SS assembly. Recent evidence demonstrates that $\mathrm{ClpV}$ is responsible for ATP-driven remodeling of VipA/VipB tubules (Bönemann et al. 2009) and may be required to ensure their proper assembly into a phage tail sheath-like structure (Bönemann et al. 2010).

What does the T6SS secrete? It should be noted that many T6SS loci include genes that are not considered essential for T6SS function. A few of these genes encode secreted proteins, and they appear to be restricted to very few bacterial genomes. For example, E. tarda evpP encodes a secreted protein and has orthologs in only two other genomes - those of the fish patho- gen E. ictaluri and the marine bacterium Vibrio MED222 (Wang et al. 2009; Zheng and Leung 2007). EvpP was shown to interact with Hcp and is involved in invasion of fish, but its specific function remains unknown. Likewise, the AaiC protein has no sequenced homologs, has unknown function, and is secreted by the enteroaggregative Escherichia coli T6SS (Dudley et al. 2006). P. aeruginosa Tse2 is the best characterized T6SS effector, and it will be described in detail below. No other distinct T6SS effectors have been identified to date. However, the T6SS loci in several bacterial genomes encode "evolved" VgrG proteins that may act both as tail-spike components of the T6SS machinery and as the proteins destined to effect a response in target cells. Evolved VgrG proteins carry extended C-terminal regions, which often contain one of several interesting effector domains likely to function in the cytoplasm of a target cell. Putative functions assigned to some of the evolved VgrG C-terminal domains include peptidoglycan binding, metalloprotease activity, mannose binding, pe/ppe protein activity, and adhesin activity (Pukatzki et al. 2007). Secretion and C-terminal domain functionality have been demonstrated for evolved VgrG proteins from $V$. cholerae and the human pathogen Aeromonas hydrophila. The C-terminal extension of $V$. cholerae VgrG1 carries an RtxA toxin domain and is translocated into target cells in which it crosslinks actin (Ma

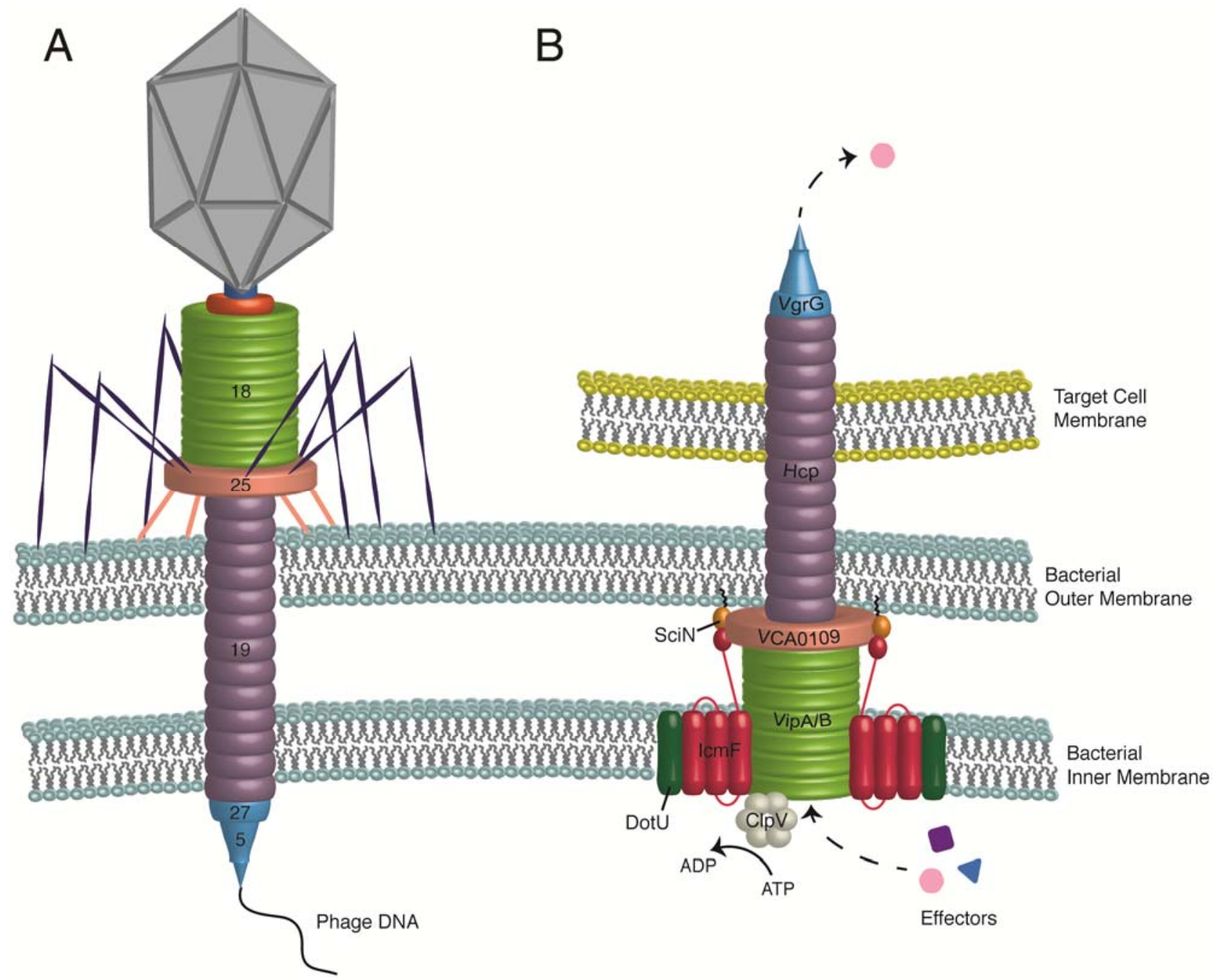

Fig. 1. Schematic drawings of the bacteriophage T4 and putative type VI secretion system (T6SS) injection machines. A. Bacteriophage T4 molecular architecture has been studied extensively and is well-established (Mesyanzhinov et al. 2004). Components are labeled with their gene product numbers as described in text. B. The T6SS model is based on predicted subcellular localizations of proteins and on the homology between T6SS and phage protein sequences. T6SS proteins with homology to phage proteins are colored the same as their T4 phage counterparts. 
and Mekalanos 2010; Ma et al. 2009b). VgrG1 from Aeromonas hydrophila induces host-cell toxicity through the ADP-ribosylating activity of its C-terminal VIP-2 domain (Suarez et al. 2010).

\section{A versatile tool.}

Mounting evidence implicates the T6SS as a virulence factor (Pilatz et al. 2006; Potvin et al. 2003; Pukatzki et al. 2007; Schell et al. 2007; Shalom et al. 2007; Suarez et al. 2010; Wang et al. 2009). However, T6SS loci are highly conserved in bacterial genomes, including those of nonpathogenic species. This suggests that bacteria may employ the T6SS in situations other than pathogenic interactions with a host. Support for this hypothesis comes from several studies dating back to the 1997 discovery of the $R$. leguminosarum imp locus (Roest et al. 1997). Nitrogen-fixing $R$. leguminosarum forms nodules on the roots of leguminous plants during highly specific endosymbiotic interactions. $R$. leguminosarum sp. strain RBL5523 nodulates the roots of both vetch and pea plants, but RBL5523 nitrogen-fixation activity is limited to vetch. Bladergroen and associates (2003) found that deletion of impJ allowed RBL5523 to form nitrogen fixation-competent nodules on pea. The specific function of ImpJ has not yet been defined, but it is now known to be a conserved, essential component of the T6SS. These results suggest that the $R$. leguminosarum T6SS is involved in host specificity. The T6SS may also play a role in limiting the virulence of certain pathogens. In 2005, Parsons and Heffron showed that the Salmonella enterica serovar Typhimurium sciS gene (now recognized as an ortholog of T6SS $i c m F$ ) limits replication in macrophages and decreases the pathogen's virulence in a mouse model (Parsons and Heffron 2005). In the context of the $S$. enterica-host interaction, the T6SS may function to limit acute virulence activities, thereby contributing to long-term colonization of the host. A similar observation has been made for the interaction between the symbiont Helicobacter hepaticus and the gastrointestinal tracts of rodents. Chow and Mazmanian (2010) demonstrated that colonization of mouse colon and invasion of intestinal epithelial cells by $H$. hepaticus are enhanced upon mutation of either icmF or hcp. Furthermore, H. hepaticus T6SS mutants induced stronger inflammatory responses than did the wildtype strain, leading the authors to propose that the T6SS may function to limit inflammation, thereby providing a balanced relationship between bacterium and host (Chow and Mazmanian 2010).

The T6SS has also been characterized as a mediator of competitive interbacterial interactions. The only T6SS effector characterized to date is the $P$. aeruginosa protein Tse2, which is apparently targeted toward other bacterial cells as the toxin component of a toxin-immunity system (Hood et al. 2010). Bacterial cells lacking Tsi2, the cognate immunity protein to Tse2, suffer arrested growth when Tse2 is expressed intracellularly. Tse 2 delivery into bacterial cells has not been demonstrated, but several lines of circumstantial evidence indicate that Tse 2 is injected by the $P$. aeruginosa T6SS into the cytosol of bacterial competitors: i) tse 2 and T6SS genes are coregulated, ii) Tse 2 secretion in culture requires intact $\operatorname{clp} V$, iii) contact between Tse 2 donor and recipient cells is required for toxic effect, and iv) physical interactions between Tse 2 and Tsi2 were demonstrated in vitro (Hood et al. 2010). MacIntyre and associates (2010) recently showed that, like that of $P$. aeruginosa, the $V$. cholerae T6SS also exhibits antimicrobial properties. In predator-prey experiments, $V$. cholerae sp. strain V52 caused up to a 100,000-fold reduction in culturable Escherichia coli, and this virulence was both T6SS- and cell contact-dependent (MacIntyre et al. 2010). V. cholerae V52 also exhibited T6SS-dependent virulence toward $S$. enterica serovar
Typhimurium and Citrobacter rodentium but not $P$. aeruginosa, indicating that its T6SS antimicrobial effects are limited to a specific range of prey (MacIntyre et al. 2010). Perhaps the most intriguing T6SS situation is that of Burkholderia thailandensis. B. thailandensis is a close, weakly pathogenic, relative of Burkholderia pseudomallei, a versatile pathogen of humans, animals, and plants (Brett et al. 1998; Lee et al. 2010). The $B$. thailandensis and $B$. pseudomallei genomes carry five and six distinct T6SS loci, respectively, which may reflect their versatility as pathogens and their ubiquity in the environment. Schwarz and associates (2010) found that T6SS-5, a T6SS locus shared by $B$. thailandensis and B. pseudomallei, is required for virulence in a mouse acute pneumonia model. Specifically, T6SS-5 was involved in bacterial survival of innate immune responses (Schwarz et al. 2010). Interestingly, the other four shared Burkholderia T6SS loci were dispensable for virulence in the mouse model. The authors also found that $B$. thailandensis T6SS-1 is involved in cell-contact dependent interbacterial interactions. T6SS-1-deficient $B$. thailandensis cells were susceptible to cell contact-induced stasis in growth competition assays with Pseudomonas fluorescens, Pseudomonas putida, and Serratia proteamaculans (Schwarz et al. 2010). In addition, T6SS-1 was required for $B$. thailandensis persistence in mixed biofilms with $P$. putida. Taken together, these results indicate that Burkholderia spp. have adapted multiple T6SS loci for distinct purposes, including interactions with hosts and with other bacteria. This is likely the case for other gram-negative bacteria, as many species carry more than one T6SS locus in their genomes (Bingle et al. 2008; Boyer et al. 2009).

Certain bacterial species appear to have adapted T6SS components for use in cooperative social behaviors. For example, the human pathogen Proteus mirabilis is very efficient at swarming motility and, when grown on agar media, Proteus mirabilis colonies from the same isolate rapidly merge (Dienes 1946). However, when inoculated on the same plate, different Proteus mirabilis isolates exhibit a swarming pattern that is limited to distinct colonies separated by clearly visible boundaries. Proteus mirabilis isolates are able to distinguish self from nonself, and this social behavior is mediated, in part, by the vgrG homolog idsB (Gibbs et al. 2008). A formal link between the idsB phenotype and Proteus mirabilis secretion activity has not yet been made, but the possibility that the T6SS is involved in bacterial self-identity is intriguing. The T6SS may also be involved in the social life of Myxococcus xanthus. M. xanthus is a highly social saprophyte that lives in the soil, decomposing dead organic matter and preying on other microorganisms. Myxobacteria carry out complex lifecycles that culminate, during times of starvation, in the formation of environmentally resistant fruiting bodies (Goldman et al. 2006). The authors of a recent review of $M$. xanthus extracellular biology described unpublished data indicating that deletion of an $M$. xanthus T6SS locus rendered the bacteria unable to form mature fruiting bodies despite having no obvious growth or motility defects (Konovalova et al. 2010). M. xanthus fruiting body formation is a highly coordinated social activity, and it will be interesting to learn more about T6SS involvement in this phenomenon. Certain T6SS effectors may function as bacterial social cues instructing recipient individuals to modulate their behaviors for the common good. The T6SS appears to be a versatile tool that has been adapted to a range of fitness activities including the facilitation of commensal or pathogenic relationships between bacteria and eukaryotes as well as cooperative or competitive interactions between bacteria.

\section{Plant-associated bacteria and their T6SS.}

Studies of human and animal pathogens have contributed the most to our current understanding of T6SS structure and 
function. However, T6SS genes are ever-present in the genomes of plant pathogens, symbionts, and other plant-associated bacteria. A recent survey of 400 bacterial genome sequences identified T6SS loci in the genomes of more than 100 bacteria, including 13 plant pathogens, four symbionts, and three plant growth-promoting rhizobacteria (Bingle et al. 2008). Table 1 includes an inventory of T6SS loci identified in plant-associated bacterial genomes. For a few of these plant-associated bacteria, including the pathogens Agrobacterium tumefaciens, Pectobacterium atrosepticum, and Pseudomonas syringae, T6SS functionality has been demonstrated.

Transcriptome profiling led to the discovery of a T6SS locus in the genome of A. tumefaciens C58, the causal agent of crown gall disease. Expression of genes in the A. tumefaciens imp locus was induced when cells were grown under mildly acidic conditions mimicking the rhizosphere (Yuan et al. 2008). Functionality of the A. tumefaciens T6SS was demonstrated through a study of the A. tumefaciens secretome; Hcp was expressed and secreted under various growth conditions and, interestingly, deletion of $h c p$ resulted in reduced tumorigenesis efficiency on potato tuber disks (Wu et al. 2008). Transcriptome profiling also led to the identification of a Pectobacterium atrosepticum T6SS locus, which appears to be regulated by quorum sensing. T6SS gene expression was reduced in a Pectobacterium atrosepticum derivative with a deletion of expI, a gene responsible for $\mathrm{N}$-(3-oxohexanoyl)-L-homoserine lactone synthesis (Liu et al. 2008). In the same study, Pectobacterium atrosepticum derivatives with deletions in either ECA3438 (impJ) or ECA3444 (vipB) exhibited slightly reduced virulence in potato stems and tubers. Mattinen and associates (2008) demonstrated that expression of the Pectobacterium atrosepticum T6SS was induced upon exposure to potato tuber extracts. In their study, however, mutation of the Pectobacterium atrosepticum ECA3432 ( $\mathrm{icmF}$ ) gene resulted in increased potato tuber maceration, which suggests that the Pectobacterium atrosepticum T6SS may participate in antipathogenesis activity.

P. syringae pv. syringae $\mathrm{B} 728 \mathrm{a}$ is an opportunistic pathogen of bean plants, in which it resides primarily as an epiphyte. Records and Gross (2010a and b) recently demonstrated that the B728a genome encodes a functional T6SS. B728a secretion of Hcp is dependent on functional $\mathrm{ClpV}$ and is regulated by the sensor kinases RetS, LadS, and GacS (Records and Gross 2010b). In this study, a B728a $c l p V$ mutant produced disease symptoms on bean similar to those caused by wildtype B728a. The lack of obvious phenotype in planta may indicate that the T6SS functions in other aspects of B728a fitness-possibly in host specificity or intermicrobial interactions. As a resident of the phyllosphere, B728a comes into contact with other microbes, including bacteria competing for resources, protozoa grazing for bacteria as food, or even microbes that may serve as nutrition for B728a. Wichmann and associates (2008) showed that the B728a protein phcA induces programmed cell death in the filamentous fungus Neurospora crassa, which B728a is able to utilize as a nutrient source. The PhcA protein sequence contains no $\mathrm{N}$ - or $\mathrm{C}$-terminal signal peptide, leading to speculation that $\mathrm{PhcA}$ may be secreted by the B728a T6SS (Wichmann et al. 2008). While a role for the T6SS in B728a-fungal interactions has not yet been demonstrated, a brief report from the Kobayashi laboratory offers some exciting support for this possibility (Patel et al. 2009). Patel and associates (2009) found that an hcp mutation in the genome of Lysobacter enzymogenes reduced the bacterium's ability to colonize fungal hosts. L. enzymogenes is a pathogen of lower eukaryotes, including nematodes, fungi, and lower plants. The bacterium is used as biocontrol agent to limit a wide range of fungal plant diseases and has the potential to reduce bacterial disease as well (Patel et al. 2009; Qian et al. 2010).
T6SS gene expression has been observed for other plant growth-promoting rhizobacteria. $P$. fluorescens strains are widely used as biocontrol agents capable of suppressing various soilborne fungal and nematode diseases. For example, $P$. fluorescens Pf29Arp is an efficient suppressor of take-all disease of wheat (Chapon et al. 2002). Analysis of the Pf29Arp transcriptome revealed that $v g r G$ expression is induced when the bacteria are exposed to necrotic conditions associated with the colonization of wheat roots by the take-all fungus Gaeumannomyces graminis var. tritici (Barret et al. 2009). Microarray studies of the $P$. fluorescens Pf-5 genome revealed that, like that of $P$. syringae B728a, the Pf-5 T6SS locus is regulated by global regulator GacA (Hassan et al. 2010; Records and Gross 2010a). Mutation of gacA resulted in significantly reduced expression of 14 genes in the Pf-5 T6SS locus. Functional studies are needed to augment the $P$. fluorescens transcriptome data so that we may begin to understand T6SS influence on the ecology and social activities of this important biocontrol agent.

\section{Concluding remarks.}

With its role in virulence, prevalence among gram-negative bacterial genomes, and predicted phage-like structure, the T6SS has become an enticing object of research. In the four years since the first descriptions of a T6SS, data regarding its structure and function have accumulated quickly, but many questions remain. We have learned a great deal from studies of the T6SS as it relates to bacterial infection in humans. It is evident, though, that to fully understand the T6SS, future inquiries must be conducted in the context of environmental fitness. Continued efforts to elucidate T6SS involvement in interbacterial interactions promise to reveal new insights into social behaviors associated with biofilm formation on surfaces, polymicrobial infections in humans and animals, and communication within heterogeneous microbial communities on plant tissues. Likewise, identification of pathogen-specific T6SS components

Table 1. Type VI secretion systems (T6SS) of plant-associated bacteria.

\begin{tabular}{|c|c|}
\hline Bacterial strain & No. of loci ${ }^{a}$ \\
\hline \multicolumn{2}{|l|}{ Pathogens } \\
\hline Acidovorax avenae subsp. citrulli AAC00-1 & 1 \\
\hline Agrobacterium tumefaciens str. C58 & 1 \\
\hline Burkholderia cepacia AMMD & 2 \\
\hline B. vietnamensis $\mathrm{G} 4$ & 3 \\
\hline Erwinia pyrifoliae DSM $12163 \mathrm{~T}$ & 2 \\
\hline Pectobacterium atrosepticum & 1 \\
\hline Pseudomonas syringae pv. oryzae 1-6 & $2^{\mathrm{b}}$ \\
\hline P. syringae pv. phaseolicola $1448 \mathrm{~A}$ & 1 \\
\hline P. syringae pv. syringae $\mathrm{B} 728 \mathrm{a}$ & 1 \\
\hline P. syringae pv. tabaci ATCC 11528 & $2^{\mathrm{b}}$ \\
\hline P. syringae pv. tomato DC 3000 & 2 \\
\hline P. syringae pv. tomato $\mathrm{T} 1$ & $2^{\mathrm{b}}$ \\
\hline Ralstonia solanacearum & 1 \\
\hline Xanthomonas axonopodis pv. citri 306 & 2 \\
\hline Xanthomonas campestris pv. vesicatoria $85-10$ & 3 \\
\hline Xanthomonas oryzae pv. oryzae KACC10331 & 2 \\
\hline Xanthomonas oryzae pv. oryzae MAFF311018 & 2 \\
\hline \multicolumn{2}{|l|}{ Plant growth-promoting rhizobacteria } \\
\hline Lysobacter enzymogenes & $2^{\mathrm{c}}$ \\
\hline P. fluorescens Pf-5 & 1 \\
\hline P. fluorescens $\mathrm{PfO}-1$ & 2 \\
\hline P. putida $\mathrm{KT} 2440$ & 3 \\
\hline \multicolumn{2}{|l|}{ Symbionts } \\
\hline Azoarcus sp. strain $\mathrm{BH} 72$ & 2 \\
\hline Bradyrhizobium japonicum USDA 110 & 1 \\
\hline Mesorhizobium loti MAFF303099 & 1 \\
\hline Rhizobacterium leguminosarum bv. viciae 3841 & 1 \\
\hline Serratia proteamaculans & 1 \\
\hline
\end{tabular}

\footnotetext{
${ }^{a}$ Number of T6SS loci in the genome (Bingle et al. 2008).

${ }^{\mathrm{b}}$ Loci identified by Sarris and associates (2010).

${ }^{c}$ Loci identified by Patel and associates (2009).
} 
and substrates may reveal novel targets for treatment of bacterial disease.

\section{ACKNOWLEDGMENTS}

Thanks to V. T. Lee (University of Maryland) and D. C. Gross (Texas A\&M University) for their critical reviews of this manuscript.

\section{LITERATURE CITED}

Aksyuk, A. A., Leiman, P. G., Kurochkina, L. P., Shneider, M. M., Kostyuchenko, V. A., Mesyanzhinov, V. V., and Rossmann, M. G. 2009. The tail sheath structure of bacteriophage T4: A molecular machine for infecting bacteria. EMBO (Eur. Mol. Biol. Organ.) J. 28:821-829.

Alvarez-Martinez, C. E., and Christie, P. J. 2009. Biological diversity of prokaryotic type IV secretion systems. Microbiol. Mol. Biol. Rev. 73:775-808.

Aschtgen, M. S., Bernard, C. S., De Bentzmann, S., Lloubès, R., and Cascales, E. 2008. SciN is an outer membrane lipoprotein required for type VI secretion in enteroaggregative Escherichia coli. J. Bacteriol. 190:7523-7531.

Aubert, D., MacDonald, D. K., and Valvano, M. A. 2010. BcsK $\mathrm{C}_{\mathrm{C}}$ is an essential protein for the Type VI secretion system activity in Burkholderia cenocepacia that forms an outer membrane complex with BcsL $_{\mathrm{B}}$. J. Biol. Chem. 285:35988-35998.

Ballister, E. R., Lai, A. H., Zuckermann, R. N., Cheng, Y., and Mougous, J. D. 2008. In vitro self-assembly of tailorable nanotubes from a simple protein building block. Proc. Natl. Acad. Sci. U.S.A. 105:3733-3738.

Barret, M., Frey-Klett, P., Guillerm-Erckelboudt, A.-Y., Boutin, M., Guernec, G., and Sarniguet, A. 2009. Effect of wheat roots infected with the pathogenic fungus Gaeumannomyces graminis var. tritici on gene expression of the biocontrol bacterium Pseudomonas fluorescens Pf29Arp. Mol. Plant-Microbe Interact. 22:1611-1623.

Bingle, L. E., Bailey, C. M., and Pallen, M. J. 2008. Type VI secretion: A beginner's guide. Curr. Opin. Microbiol. 11:3-8.

Bladergroen, M. R., Badelt, K., and Spaink, H. P. 2003. Infection-blocking genes of a symbiotic Rhizobium leguminosarum strain that are involved in temperature-dependent protein secretion. Mol. Plant-Microbe Interact. 16:53-64.

Bönemann, G., Pietrosiuk, A., and Mogk, A. 2010. Tubules and donuts: A type VI secretion story. Mol. Microbiol. 76:815-821.

Bönemann, G., Pietrosiuk, A., Diemand, A., Zentgraf, H., and Mogk, A. 2009. Remodelling of VipA/VipB tubules by ClpV-mediated threading is crucial for type VI protein secretion. EMBO (Eur. Mol. Biol. Organ.) J. 28:315-325.

Boyer, F., Fichant, G., Berthod, J., Vandenbrouck, Y., and Attree, I. 2009. Dissecting the bacterial type VI secretion system by a genome wide in silico analysis: What can be learned from available microbial genomic resources? BMC Genomics 10:104

Brett, P. J., DeShazer, D., and Woods, D. E. 1998. Burkholderia thailandensis sp. nov., a Burkholderia pseudomallei-like species. Int. J. Syst. Bacteriol. 48 Pt 1:317-320.

Cascales, E. 2008. The type VI secretion toolkit. EMBO (Eur. Mol. Biol. Organ.) Rep. 9:735-741.

Chapon, A., Guillerm, A. Y., Delalande, L., Lebreton, L., and Sarniguet, A. 2002. Dominant colonisation of wheat roots by Pseudomonas fluorescens Pf29A and selection of indigenous microflora in the presence of the take-all fungus. Eur. J. Plant Pathol. 108:449-459.

Chow, J., and Mazmanian, S. K. 2010. A pathobiont of the microbiota balances host colonization and intestinal inflammation. Cell Host Microbe 7:265-276.

Cianciotto, N. P. 2005. Type II secretion: A protein secretion system for all seasons. Trends Microbiol. 13:581-588.

Cornelis, G. R. 2006. The type III secretion injectisome. Nat. Rev. Microbiol. 4:811-825.

Das, S., Chakrabortty, A., Banerjee, R., and Chaudhuri, K. 2002. Involvement of in vivo induced icmF gene of Vibrio cholerae in motility, adherence to epithelial cells, and conjugation frequency. Biochem. Biophys. Res. Commun. 295:922-928.

Delepelaire, P. 2004. Type I secretion in gram-negative bacteria. Biochim. Biophys. Acta 1694:149-161.

Dienes, L. 1946. Reproductive processes in Proteus cultures. Proc. Soc. Exp. Biol. Med. 63:265-270.

Dudley, E. G., Thomson, N. R., Parkhill, J., Morin, N. P., and Nataro, J. P. 2006. Proteomic and microarray characterization of the AggR regulon identifies a pheU pathogenicity island in enteroaggregative Escherichia coli. Mol. Microbiol. 61:1267-1282.

Duong, F., Lazdunski, A., and Murgier, M. 1996. Protein secretion by het- erologous bacterial ABC-transporters: The C-terminus secretion signal of the secreted protein confers high recognition specificity. Mol. Microbiol. 21:459-470.

Gibbs, K. A., Urbanowski, M. L., and Greenberg, E. P. 2008. Genetic determinants of self identity and social recognition in bacteria. Science 321:256-259.

Goldman, B. S., Nierman, W. C., Kaiser, D., Slater, S. C., Durkin, A. S., Eisen, J. A., Ronning, C. M., Barbazuk, W. B., Blanchard, M., Field, C., Halling, C., Hinkle, G., Iartchuk, O., Kim, H. S., Mackenzie, C., Madupu, R., Miller, N., Shvartsbeyn, A., Sullivan, S. A., Vaudin, M., Wiegand, R., and Kaplan, H. B. 2006. Evolution of sensory complexity recorded in a myxobacterial genome. Proc. Natl. Acad. Sci. U.S.A. 103:15200-15205.

Hassan, K. A., Johnson, A., Shaffer, B. T., Ren, Q., Kidarsa, T. A., Elbourne, L. D. H., Hartney, S., Duboy, R., Goebel, N. C., Zabriskie, T. M., Paulsen, I. T., and Loper, J. E. 2010. Inactivation of the GacA response regulator in Pseudomonas fluorescens Pf-5 has far-reaching transcriptomic consequences. Environ. Microbiol. 12:899-915.

Henderson, I. R., Navarro-Garcia, F., Desvaux, M., Fernandez, R. C., and Ala'Aldeen, D. 2004. Type V protein secretion pathway: The autotransporter story. Microbiol. Mol. Biol. Rev. 68:692-744.

Hood, R. D., Singh, P., Hsu, F., Güvener, T., Carl, M. A., Trinidad, R. R. S., Silverman, J. M., Ohlson, B. B., Hicks, K. G., Plemel, R. L., Li, M. Schwarz, S., Wang, W. Y., Merz, A. J., Goodlett, D. R., and Mougous, J. D. 2010. A type VI secretion system of Pseudomonas aeruginosa targets a toxin to bacteria. Cell Host Microbe 7:25-37.

Jobichen, C., Chakraborty, S., Li, M., Zheng, J., Joseph, L., Mok, Y.-K., Leung, K. Y., and Sivaraman, J. 2010. Structural basis for the secretion of EvpC: A key type VI secretion system protein from Edwardsiella tarda. PLoS ONE 5:e12910. Published online.

Konovalova, A., Petters, T., and Søgaard-Andersen, L. 2010. Extracellular biology of Myxococcus xanthus. FEMS (Fed. Eur. Microbiol. Soc.) Microbiol. Rev. 34:89-106.

Lee, Y. H., Chen, Y., Ouyang, X., and Gan, Y.-H. 2010. Identification of tomato plant as a novel host model for Burkholderia pseudomallei. BMC Microbiol. 10:28.

Leiman, P. G., Chipman, P. R., Kostyuchenko, V. A., Mesyanzhinov, V. V., and Rossmann, M. G. 2004. Three-dimensional rearrangement of proteins in the tail of bacteriophage T4 on infection of its host. Cell 118:419-429.

Leiman, P. G., Basler, M., Ramagopal, U. A., Bonanno, J. B., Sauder, J. M., Pukatzki, S., Burley, S. K., Almo, S. C., and Mekalanos, J. J. 2009. Type VI secretion apparatus and phage tail-associated protein complexes share a common evolutionary origin. Proc. Natl. Acad. Sci. U.S.A. 106:4154-4159.

Liu, H., Coulthurst, S. J., Pritchard, L., Hedley, P. E., Ravensdale, M., Humphris, S., Burr, T., Takle, G., Brurberg, M. B., Birch, P. R. J., Salmond, G. P. C., and Toth, I. K. 2008. Quorum sensing coordinates brute force and stealth modes of infection in the plant pathogen Pectobacterium atrosepticum. PLoS Pathog. 4:e1000093. Published online.

Ma, A. T., and Mekalanos, J. J. 2010. In vivo actin cross-linking induced by Vibrio cholerae type VI secretion system is associated with intestinal inflammation. Proc. Natl. Acad. Sci. U.S.A. 107:4365-4370.

Ma, L.-S., Lin, J.-S., and Lai, E.-M. 2009a. An IcmF family protein, $\operatorname{ImpL}_{\mathrm{M}}$, is an integral inner membrane protein interacting with $\operatorname{ImpK}_{\mathrm{L}}$, and its walker a motif is required for type VI secretion system-mediated Hcp secretion in Agrobacterium tumefaciens. J. Bacteriol. 191:43164329.

Ma, A. T., McAuley, S., Pukatzki, S., and Mekalanos, J. J. 2009b. Translocation of a Vibrio cholerae type VI secretion effector requires bacterial endocytosis by host cells. Cell Host Microbe 5:234-243.

MacIntyre, D. L., Miyata, S. T., Kitaoka, M., and Pukatzki, S. 2010. The Vibrio cholerae type VI secretion system displays antimicrobial properties. Proc. Natl. Acad. Sci. U. S. A. 107:19520-19524.

Mattinen, L., Nissinen, R., Riipi, T., Kalkkinen, N., and Pirhonen, M. 2007. Host-extract induced changes in the secretome of the plant pathogenic bacterium Pectobacterium atrosepticum. Proteomics 7:3527-3537.

Mesyanzhinov, V. V., Leiman, P. G., Kostyuchenko, V. A., Kurochkina, L. P., Miroshnikov, K. A., Sykilinda, N. N., and Shneider, M. M. 2004 Molecular architecture of bacteriophage T4. Biochemistry (Mosc.) 69:1190-1202.

Mougous, J. D., Cuff, M. E., Raunser, S., Shen, A., Zhou, M., Gifford, C. A., Goodman, A. L., Joachimiak, G., Ordoñez, C. L., Lory, S., Walz, T., Joachimiak, A., and Mekalanos, J. J. 2006. A virulence locus of Pseudomonas aeruginosa encodes a protein secretion apparatus. Science 312:1526-1530.

Parsons, D. A., and Heffron, F. 2005. sciS, an icmF homolog in Salmonella enterica serovar Typhimurium, limits intracellular replication and decreases virulence. Infect. Immun. 73:4338-4345.

Patel, N., Blackmoore, M., Hillman, B., and Kobayashi, D. 2009. Evi- 
dence for the role of Type VI secretion during Lysobacter enzymogenes pathogenesis of fungal hosts. Phytopathology 99:100-101.

Pell, L. G., Kanelis, V., Donaldson, L. W., Howell, P. L., and Davidson, A. R. 2009. The phage lambda major tail protein structure reveals a common evolution for long-tailed phages and the type VI bacterial secretion system. Proc. Natl. Acad. Sci. U.S.A. 106:4160-4165.

Pilatz, S., Breitbach, K., Hein, N., Fehlhaber, B., Schulze, J., Brenneke, B., Eberl, L., and Steinmetz, I. 2006. Identification of Burkholderia pseudomallei genes required for the intracellular life cycle and in vivo virulence. Infect. Immun. 74:3576-3586.

Potvin, E., Lehoux, D. E., Kukavica-Ibrulj, I., Richard, K. L., Sanschagrin, F., Lau, G. W., and Levesque, R. C. 2003. In vivo functional genomics of Pseudomonas aeruginosa for high-throughput screening of new virulence factors and antibacterial targets. Environ. Microbiol. 5:12941308.

Pukatzki, S., Ma, A. T., Revel, A. T., Sturtevant, D., and Mekalanos, J. J. 2007. Type VI secretion system translocates a phage tail spike-like protein into target cells where it cross-links actin. Proc. Natl. Acad. Sci. U.S.A. 104:15508-15513.

Pukatzki, S., Ma, A. T., Sturtevant, D., Krastins, B., Sarracino, D., Nelson, W. C., Heidelberg, J. F., and Mekalanos, J. J. 2006. Identification of a conserved bacterial protein secretion system in Vibrio cholerae using the Dictyostelium host model system. Proc. Natl. Acad. Sci. USA 103:1528-1533.

Qian, G.-L., Fan, J.-Q., Chen, D.-F., Kang, Y.-J., Han, B., Hu, B.-S., and Liu, F.-Q. 2010. Reducing Pectobacterium virulence by expression of an $N$-acyl homoserine lactonase gene $\mathrm{P}_{l p p}$-aiiA in Lysobacter enzymogenes strain $\mathrm{OH} 11$. Biol. Control 52:17-23.

Rao, P. S. S., Yamada, Y., Tan, Y. P., and Leung, K. Y. 2004. Use of proteomics to identify novel virulence determinants that are required for Edwardsiella tarda pathogenesis. Mol. Microbiol. 53:573-586.

Records, A. R., and Gross, D. C. 2010a. Sensor kinases RetS and LadS regulate Pseudomonas syringae type VI secretion and virulence factors. J. Bacteriol. 192:3584-3596.

Records, A. R., and Gross, D. C. 2010b. Sensor kinases RetS and LadS regulate Pseudomonas syringae type VI secretion and virulence factors. J Bacteriol 192:3584-3596.

Roest, H. P., Mulders, I. H. M., Spaink, H. P., Wijffelman, C. A., and Lugtenberg, B. J. J. 1997. A Rhizobium leguminosarum biovar trifolii locus not localized on the sym plasmid hinders effective nodulation on plants of the pea cross-inoculation group. Mol. Plant-Microbe Interact. 10:938-941.

Sarris, P. F., Skandalis, N., Kokkinidis, M., and Panopoulos, N. J. 2010. In silico analysis reveals multiple putative type VI secretion systems and effector proteins in Pseudomonas syringae pathovars. Mol. Plant Pathol. 11:795-804

Schell, M. A., Ulrich, R. L., Ribot, W. J., Brueggemann, E. E., Hines, H.
B., Chen, D., Lipscomb, L., Kim, H. S., Mrázek, J., Nierman, W. C and DeShazer, D. 2007. Type VI secretion is a major virulence determinant in Burkholderia mallei. Mol. Microbiol. 64:1466-1485.

Schlieker, C., Zentgraf, H., Dersch, P., and Mogk, A. 2005. ClpV, a unique Hsp100/Clp member of pathogenic proteobacteria. Biol. Chem. 386:1115-1127.

Schwarz, S., West, T. E., Boyer, F., Chiang, W.-C., Carl, M. A., Hood, R. D., Rohmer, L., Tolker-Nielsen, T., Skerrett, S. J., and Mougous, J. D. 2010. Burkholderia type VI secretion systems have distinct roles in eukaryotic and bacterial cell interactions. PLoS Pathog. 6. Published online.

Sexton, J. A., Miller, J. L., Yoneda, A., Kehl-Fie, T. E., and Vogel, J. P. 2004. Legionella pneumophila DotU and $\mathrm{IcmF}$ are required for stability of the Dot/Icm complex. Infect. Immun. 72:5983-5992.

Shalom, G., Shaw, J. G., and Thomas, M. S. 2007. In vivo expression technology identifies a type VI secretion system locus in Burkholderia pseudomallei that is induced upon invasion of macrophages. Microbiology 153:2689-2699.

Suarez, G., Sierra, J. C., Erova, T. E., Sha, J., Horneman, A. J., and Chopra, A. K. 2010. A type VI secretion system effector protein, VgrG1, from Aeromonas hydrophila that induces host cell toxicity by ADP ribosylation of actin. J. Bacteriol. 192:155-168.

VanRheenen, S. M., Dumenil, G., and Isberg, R. R. 2004. IcmF and DotU are required for optimal effector translocation and trafficking of the Legionella pneumophila vacuole. Infect. Immun. 72:5972-5982.

Wang, X., Wang, Q., Xiao, J., Liu, Q., Wu, H., Xu, L., and Zhang, Y. 2009. Edwardsiella tarda T6SS component evpP is regulated by esrB and iron, and plays essential roles in the invasion of fish. Fish Shellfish Immunol. 27:469-477.

Wichmann, G., Sun, J., Dementhon, K., Glass, N. L., and Lindow, S. E. 2008. A novel gene, phcA from Pseudomonas syringae induces programmed cell death in the filamentous fungus Neurospora crassa. Mol. Microbiol. 68:672-689.

Williams, S. G., Varcoe, L. T., Attridge, S. R., and Manning, P. A. 1996. Vibrio cholerae Hcp, a secreted protein coregulated with HlyA. Infect. Immun. 64:283-289.

Wu, H.-Y., Chung, P.-C., Shih, H.-W., Wen, S.-R., and Lai, E.-M. 2008 Secretome analysis uncovers an Hcp-family protein secreted via a type VI secretion system in Agrobacterium tumefaciens. J. Bacteriol. 190:2841-2850.

Yuan, Z.-C., Liu, P., Saenkham, P., Kerr, K., and Nester, E. W. 2008. Transcriptome profiling and functional analysis of Agrobacterium tumefa ciens reveals a general conserved response to acidic conditions ( $\mathrm{pH} 5.5)$ and a complex acid-mediated signaling involved in Agrobacteriumplant interactions. J. Bacteriol. 190:494-507.

Zheng, J., and Leung, K. Y. 2007. Dissection of a type VI secretion system in Edwardsiella tarda. Mol. Microbiol. 66:1192-1206. 